\title{
INTRODUCCIÓN \\ Diversidad en el campo \\ de la Comunicación
}

La introducción sirve de lectura de inicio al XV Anuario de Investigación de la Comunicación CONEICC (xV AIC CONEICC). El XV AIC CONEICC es un esfuerzo ininterrumpido a lo largo de los últimos 15 de los 32 años de la vida que tiene el Consejo Nacional para la Enseñanza y la Investigación de las Ciencias de la Comunicación. Esta publicación periódica constituye el foro impreso más importante del Consejo y en el momento actual se encuentra en vías de transformación. A petición de las instituciones integrantes del Consejo, se han iniciado los trabajos para que el Anuario se convierta en revista científica, arbitrada y, en su momento, logre la indización, proceso que ha asumido la actual gestión.

\section{DiVERSIFICACIÓN Y MADURACIÓN EN EL CAMPO}

Los trabajos que integran el presente número del Anuario, refieren a los pensadores de la Comunicación a la variedad de tendencias, corrientes y perspectivas que aportan sus autores como contribución al debate de la misma como campo temático.

Hacia el fin de la primera década del siglo xxi, el campo de la Comunicación continúa su diversificación. Se han detectado ocho grandes áreas temáticas o tendencias que configuran hoy por hoy el espectro de la generación del conocimiento en comunicación, la enseñanza al interior de las escuelas y sus aplicaciones prácticas en América Latina: 1) tendencia que se concentra en el estudio de los medios de comunicación masiva y de las nuevas tecnologías (hipermedios), su influencia en las audiencias/usuarios y la generación de nuevas ecologías mediáticas y culturales; 2) pensamiento que sostiene que la comunicación carece 
de los componentes que hacen de ésta una ciencia en sí, razón por la cual vale la pena recurrir a otras ciencias tales como la Sociología o los enfoques culturales, ente otros, para desde ahí estudiar a la comunicación; 3) corriente que estudia los procesos culturales y dentro de éstos a la comunicación como un proceso de creación de sentido; 4) tendencia que apuesta a la construcción de la ciencia de la comunicación como un proceso en construcción: Comunicología Posible; 5) directriz que aglutina a estudiosos bajo el nombre de Laboratorio de Investigación y Desarrollo en Comunicación Compleja (LabCOMplex) que busca generar conocimiento sobre las formas en que los distintos grupos socioculturales usan y producen información y entretenimiento con las herramientas de la comunicación y las nuevas tecnologías y que considera las posibilidades de su uso para que sus usuarios tengan mayor calidad de vida y de cultura; 6) línea que centra la generación del conocimiento acerca de la comunicación desde las ciencias del lenguaje y de la semiótica; 7) corriente que busca sistematizar el conocimiento de la comunicación en América Latina, con frecuencia llamada la Escuela Latinoamericana de la Comunicación (ELACOM) y que se caracteriza por su mestizaje teórico, su hibridismo metodológico, su compromiso ético político y su dimensión extranacional; 8) tendencia reciente que sostiene que el área de la comunicación integral en las organizaciones es una disciplina en sí y que analiza los procesos de comunicación, más que en su dimensión social amplia, en tres vertientes dentro y relacionadas con las organizaciones: la comunicación interna, la corporativa y la mercadológica o publicidad.

Dada esta riqueza de tendencias y vertientes que han ampliado la gama de formas de ver y penetrar en el fenómeno comunicativo, así como de producir conocimiento para su aplicación en la práctica, hoy se puede decir que se llega a un estadio de mayor madurez en el campo.

\section{HACIA LA CONSTRUCCIÓN DE LA COMUNIDAD CIENTÍfICA DE LA COMUNICACIÓN}

El día de hoy, es posible señalar que a escasos 50 años $^{1}$ de los albores de la investigación de la comunicación en América Latina, se está llegando a estadios de mayor madurez. ¿Cómo se manifiesta esta madurez? 1) Son ya un total de 347 Programas de Comunicación ${ }^{2}$ en sus diversas modalidades que forman a

${ }^{1}$ El 9 de Octubre de 1959, se funda el Centro Internacional de Estudios Superiores de Comunicación para América Latina, (CIESPAL).

${ }^{2}$ ANUIEs, 2007, Catálogo de carreras de Licenciatura en Universidades e Institutos Tecno-

20 - María Antonieta Rebeil Corella 
más de $72,663^{3}$ estudiantes en el territorio nacional. 2) Por una parte, en el marco nacional existe una mayor proliferación de Centros de Investigación de la Comunicación ${ }^{4}$ mismos que buscan la consolidación de la teoría de la comunicación así como sistematizar las prácticas comunicacionales. 3) Por otra parte, no solamente existen más investigadores en comunicación en el territorio nacional, sino que éstos se empiezan a agrupar en redes de investigación $\left.{ }^{5} .4\right)$ Adicionalmente, los presupuestos destinados a la investigación por parte de las Instituciones de Educación Superior, observan un ascenso que se ve reflejado en el surgimiento de la figura del profesor-investigador que gana presencia en el ámbito de los recursos humanos de las universidades. 5) Además, existe, en el marco nacional, un número de investigadores reconocidos y remunerados por el Sistema Nacional de Investigadores (SNI) que asciende a $13,485^{6}$, dentro de los cuales, los investigadores SNI en ciencias sociales, son: $1,854^{7}$. 6) Por otro lado, es preciso hacer una mención especial a la proliferación de los programas de posgrado que han surgido a lo largo y ancho de México. En el año 2005, se registraron 957 Maestrías en Comunicación con diversas especialidades; cinco de ellas acreditadas por el CONACYT. ${ }^{8}$ 7) Hoy se registran 5 programas de Doctorado acreditados por CONACYT y con oferta de formación en comunicación. ${ }^{9}$

lógicos, recuperado el 19 de agosto de 2008, de http://www.anuies,mx/servicios/c_licenciatu$\mathrm{ra} /$ index2.php

${ }^{3}$ Fuentes, R, Datos de la Anuies, 2005, en J. Calles (Ed.) (2005) XII Anuario de Investigación de la Comunicación CONEICC, México: CONEICC.

${ }^{4}$ Entre los que se encuentran los siguientes: Coordinación de Investigación del Centro de Estudios en Ciencias de la Comunicación, Universidad Nacional Autónoma de México (unAM); Centro de Investigación en Comunicación e Información, Centro de Investigación en Comunicación Anáhuac-Mayab (CICAM); Centro de Investigación para la Comunicación Aplicada (CICA) Universidad Anáhuac México Norte; Coordinación de Investigación y Diseño Curricular, Universidad Latina de América; Programa Formal de Investigación en Estudios Socioculturales Instituto Tecnológico de Estudios Superiores de Occidente (ITESo); Programa Interinstitucional de Investigación en Comunicación (PIIC), Universidad del Valle de México; Proyecto Internet; Instituto Tecnológico y de Estudios Superiores de Monterrey, campus Estado de México.

${ }^{5}$ Ver página web del conEICC y las redes de investigación presentadas

${ }^{6}$ CONACYT, Base de datos del SNI, 2006.

${ }^{7}$ CONACYT, Base de datos del SNi, 2006

${ }^{8}$ Fuentes, R, Datos de la Anuies, 2005, en J. Calles (Ed.) (2005) XII Anuario de Investigación de la Comunicación CONEICC, México: CONEICC.

${ }^{9}$ Fuentes, R, Datos de la Anuies, 2005, en J. Calles (Ed.) (2005) XII Anuario de Investigación de la Comunicación CONEICC, México: CONEICC. 
De la misma manera se puede hacer mención a 8) la cantidad de revistas, tanto de divulgación como científicas, que han surgido sobre el tema de la comunicación en los últimos años. Muchas de éstas, aparecen de manera impresa y han adquirido un gran renombre en el panorama nacional ${ }^{10}$. Otras más, $987^{11}$, para hablar en cifras, surgen de manera digital en respuesta al fenómeno de la globalización del conocimiento ${ }^{12}$. Es importante señalar que cada vez éstos medios de difusión del conocimiento científico están siendo dirigidos por comunicólogos para el uso de los mismos comunicólogos.

Por otra parte, 9) se han multiplicado los foros, congresos y seminarios en los que se da a conocer y se difunde el conocimiento en investigación. En el momento actual, prácticamente todas y cada una de las universidades que tienen Escuelas de Comunicación realizan cuando menos un foro anual de comunicación; algunos de éstos con convocatoria regional, nacional e internacional.

Así mismo, se puede decir que 10) ha aumentado el valor que se le da a los nuevos y más jóvenes talentos en la investigación. Muchos de estos congresos y foros se generan por estudiantes y para los mismos estudiantes. 11) La cantidad de investigaciones en las que participan los alumnos y que encabezan jóvenes investigadores va en ascenso y actúan en calidad de semilleros de talentos.

Para dar cabida a todos estos estudiosos del campo, tanto decanos como de reciente ingreso, 12) se han fundado las asociaciones de comunicación que han ampliado los espacios para la discusión y que intentan incidir en la toma de decisiones políticas que favorecen la democratización de la comunicación en México. Estas asociaciones no solamente han tocado los ámbitos de los medios de comunicación tradicionales sino que penetran con gran insistencia en el campo de las nuevas tecnologías, sus interacciones y sus nuevas modalidades (hipermedios). Entre otras, se puede mencionar, para dar algunos ejemplos ilustrativos, a la Asociación Mexicana de Investigadores de la Comunicación (AMIC) que se fundó en 1979 y que continúa sus actividades a través de 14 Grupos de Investigación y de sus Encuentros y Publicaciones Anuales; la Asociación Mexicana de Derecho a la Información (AMEDI), que ha buscado fortalecer la discusión teórica de los derechos de los comunicadores y su incidencia en

\footnotetext{
${ }^{10}$ Entre las que se encuentran: Revista Mexicana de Comunicación, Revista Comunicación y Sociedad, Revista Iberoamericana de Comunicación, Revista Radio y Televisión Americana.

${ }^{11}$ Dato de Latindex, recuperado de http//www.latindex.unAM.mx/latindex/busquedas1/estadistica_bus.html?f=16, el 2 de junio de 2008.

${ }^{12}$ Ejemplos de esto son: Razón y Palabra, Global Media Journal en Español, SintAxis, Equis Medio Digital, En Comunicación, La Galera Net, entre muchas otras.
} 
las políticas públicas de la comunicación a fin de garantizar la equidad y la pluralidad en las comunicaciones del país; Asociación Mexicana de Internet (AMIPCI), ha dedicado sus esfuerzos a garantizar el desarrollo de Internet así como la seguridad en línea, la institucionalización de Internet y el comercio electrónico, la certificación de estándares y protocolos digitales, así como del monitoreo de la actividad en línea.

De la misma manera, 13) se pueden mencionar las asociaciones de profesionales de la comunicación y vinculadas a las organizaciones, tales como la Asociación Mexicana de Agencias de Publicidad (AMAP), Asociación Nacional de la Publicidad (ANP), Asociación Mexicana de Agencias Profesionales de Relaciones Públicas (PRORP), Asociación Mexicana de Agencias de Investigación de Mercados y Opinión Pública (AMAI) Asociación Mexicana de Comunicadores (AMCo), Confederación de la Industria de la Comunicación Mercadotécnica Сıсом. Todas éstas, en conjunto, han brindado a la profesión la viabilidad hacia su institucionalización y la apertura al diálogo productivo con los pares nacionales y en algunos casos, internacionales.

Uno de los pasos más significativos en el proceso de maduración de la comunicación en el país se refiere precisamente 14) al surgimiento del Consejo Nacional de Acreditación en Comunicación (CONAC). Este organismo, surgido en el seno del Coneicc en 2006, ha tomado un vuelo propio que le está permitiendo ser el aval de calidad de las mejores escuelas de comunicación en el país. ${ }^{13}$ Una de las cuestiones que puntualiza el ConAC para acreditar a una Escuela como una institución de calidad es precisamente la existencia de áreas o centros de investigación que aseguran la generación del conocimiento. El trayecto entre ser una institución en la cual solamente se imparten clases a una en la cual también se produce y difunde el conocimiento, es una señal clave para pasar de college a ser una verdadera Universidad.

El mismo CONEICC se encuentra realizando transformaciones importantes que dejan ver el paso de esta maduración que vive la comunidad de comunicadores. Algunos de estos procesos innovadores son: 15) la revitalización de las sesiones académicas y de discusión en el seno de las asambleas del Consejo; 16) el esfuerzo por conformar más redes de investigadores; 17) la decisión e inicio del proceso de conversión del Anuario en revista científica y arbitrada,

\footnotetext{
${ }^{13}$ Entre otras, las universidades acreditadas por el conAC en el momento actual son: Universidad Anáhuac México Norte, Universidad Iberoamericana, Universidad de Monterrey, entre otras.
} 
18) la transformación del mismo Anuario en un texto coeditado por varias instituciones miembros y que 19) sus contenidos incluyan a investigadores representativos de la mayor parte del territorio nacional; 20) los Encuentros Docentes llevados a cabo tanto en la Vocalía del Valle de México como en la del Noreste que buscan potenciar regionalmente el componente de los maestros y las mejores prácticas de los procesos de enseñanza aprendizaje de la comunicación; 21) la página web que ha ampliado la posibilidad e intercambio entre las instituciones miembros del coneicC a través del apoyo del Instituto Tecnológico y de Estudios Superiores de Monterrey (ITESM), Campus Estado de México; 22) el Centro de Documentación que, con el apoyo del Instituto Tecnológico y de Estudios Superiores de Occidente (ITESo), se ha ampliado y modernizado; 23) el Proyecto Académico del coneicc que lleva a cabo el Premio Nacional de Titulación estimulando a la investigación desde las áreas terminales de Licenciatura y Maestría.

Al unísono de los pasos que ha venido dando el CONEICC para fortalecer la disciplina de la comunicación en el país, evidentemente queda mucho por caminar. Una de las cuestiones que ocupan y preocupan a la comunidad es precisamente la construcción de plataformas para generar, difundir y posicionar el conocimiento de las ciencias de la comunicación con estándares internacionales. 24) Para ello el coneicc ha lanzado la iniciativa del Primer Seminario Nacional de Comunicación Cientifica para la Elaboración, Postulación y Evaluación de Artículos Cientificos, que se lleva acabo en seis sedes del territorio nacional convocando a los profesores-investigadores de las universidades miembro del Consejo a sumarse a este esfuerzo encaminado a la proyección internacional de la riqueza de conocimientos que se genera en el territorio nacional. Como apoyo a esta iniciativa se invitó a la Red de Revistas Científicas de América Latina, el Caribe, España y Portugal (REDALYc) que alberga la Universidad Autónoma del Estado de México (UAEM), para impartir el Seminario dado el reconocimiento que ha acumulado en el ámbito internacional.

LAS CONTRIBUCIONES DEL XV ANUARIO DE INVESTIGACIÓN DE LA COMUNICACIÓN CONEICC

La Primera Parte del Xv aic coneIcc, titulada Legislación, Ética y Medios de Comunicación en México, contiene tres artículos que tocan el tema álgido de la legislación y en consonancia con la preocupación tradicional de los pensadores mexicanos en torno a este tema que, pese a los esfuerzos, permanece sin resolver.

$24 \cdot$ María Antonieta Rebeil Corella 
María de la Luz Casas Pérez del Instituto Tecnológico y de Estudios Superiores de Monterrey, Campus Cuernavaca, abre la discusión con su trabajo: $E l$ proceso de discusión de la Ley Federal de Radio, Televisión y Telecomunicaciones en el contexto de la Teoría de la Acción Social. Casas señala que por muchos años, incluso décadas, los estudios de los medios se han concentrado en las cuestiones básicas tales como la presentación de los contenidos las audiencias. La autora sugiere que para investigar con mayor precisión a los medios de comunicación y su quehacer en la sociedad, tal vez sería más adecuado hacerlo a la luz de la Teoría de Acción Social, ya que la información que éstos transmiten se genera a partir de la creación de círculos sociales en los que los actores se desenvuelven para fortalecer la inclinación de la balanza en una dirección o en otra. Los procesos de gestión de las políticas públicas aun están lejos de ser verdaderamente comprendidos por parte de los investigadores. Sugiere la investigadora que se analice la participación de los actores sociales y cómo intervienen éstos en las discusiones para la aprobación/rechazo de las leyes que rigen el comportamiento de los medios en la sociedad.

El trabajo que presenta Javier Esteinou Madrid de la Universidad Autónoma Metropolitana, Unidad Xochimilco, se titula La Suprema Corte de Justicia y el rescate comunicativo del Estado nación: El caso de la Ley Televisa. El artículo señala las bondades de la resolución que tomó la Suprema Corte de Justicia de la Nación al no dejar pasar la decisión del Senado y de la Cámara de Diputados de dar por buena la así denominada Ley Televisa. Esteinou sustenta que ese acto coloca al Estado mexicano nuevamente en su estatus de rector de la sociedad al hacer frente a los poderes de facto que sustentan los medios de comunicación privados en la sociedad mexicana. Con este trabajo, Javier Esteinou aporta una crónica puntual de los hechos que llevaron a las Cámaras Alta y Baja a aprobar de manera expedita y sin precedentes las reformas a unas Leyes que podían haber ensanchado el desequilibrio de poder entre los medios y la sociedad. El investigador propone que la creación de una nueva Ley Federal de Radio y Televisión y de Telecomunicaciones más equitativa, puede ser motor de la democracia y la pluralidad en la Nación.

El artículo siguiente es el titulado: La comunicación como mediación ética del mundo ante la descentralización de la persona en la acción comunicativa, es un trabajo realizado por Jorge Hidalgo Toledo y José Antonio Forzán Gómez, ambos de la Universidad Anáhuac México Norte. Nos recuerdan los autores que uno de los problemas más profundos de la sociedad actual no es, como se ha querido proponer, la brecha informativa. Señalan Hidalgo y Forzán que es más grave aún 
la ausencia de una metafísica significativa que promueva la difusión de una comunidad universal de personas vinculadas por la comunicación. La comunicación centrada en la persona humana tiene una fuerza trascendental para la búsqueda del bien común, la unión y la justicia en la sociedad. Para ello una prioridad es lograr que se produzcan informaciones y contenidos mediáticos de calidad.

La segunda parte del XV Anuario de Investigación de la Comunicación CONEICC, titulada Nuevas reflexiones sobre teoría de la comunicación, contiene los artículos que elaboran y hacen avanzar la teoría (para algunos, la ciencia) de la comunicación. Inicia esta parte la aportación de Gustavo Adolfo León Duarte de la Universidad de Sonora, con el artículo: Estructura y prácticas cientificas en el pensamiento latinoamericano de la comunicación. Un acercamiento a los aportes epistemológicos de la ELACOM. En este trabajo se pretende abrir las vías de acercamiento de lo que se ha venido denominando la Escuela Latinoamericana de Comunicación. Sustenta León Duarte que más que hablar de una Escuela, es más factible hablar de un colectivo que conforma el Pensamiento Latinoamericano de la Comunicación (PLC), mismo que surge con el nacimiento del CIESPAL en Quito, Ecuador en 1959. El autor dice que ELACOM es más bien una iniciativa institucional en la búsqueda de la identidad latinoamericana en los estudios de la comunicación y que sus aportaciones han constituido los fundamentos de la fuerza del pensamiento de la región para conformar la teoría de la comunicación que responde a sus realidades socioeconómicas, políticas y culturales.

En el trabajo: La dimensión comunicativa del arte. Apuntes para un estado de la cuestión, Vivian Romeu Aldaya de la Universidad Autónoma de la Ciudad de México, demuestra que históricamente ha existido una relación entre el arte y la comunicación. Dicha conexión se crea al momento que un artista realiza una obra de arte, explicándolo mediante nodos conceptuales. Los cuatro nodos conceptuales que explica la autora, son: a) el estético-histórico, donde especialistas afirman que el arte es aquella actividad que muestra lo que sucede en la vida diaria de las personas proyectada por el artista; b) el nodo estético-cognoscitivo en el cual se explica que los receptores de la obra aparte de admirar la obra, acceden a una relación con ésta; c) el nodo estético-intuitivo que explica que las obras se desarrollan de acuerdo a la personalidad del artista; d) el cuarto nodo, estético-semiótico, donde se afirma que la admiración y comprensión del arte no sólo es cuestión de comprender sino de entender el lenguaje, ver más allá de lo que a primera vista se ve.

Mediante su trabajo, Romeu establece el entramado de relaciones que se da entre las obras de arte y el espectador, quien se va reconociendo a sí mismo, 
en éstas. De ahí que se establece un paralelismo entre comunicación y arte ya que ésta constituye, en esencia, mensajes que se comunican al receptor.

Tanius Karam Cárdenas, de la Universidad Autónoma de la Ciudad de México, en su ensayo: Carlos Monsiváis y el campo académico de la comunicación: Interacción y sentidos, propone al mismo Monsiváis como un personaje que comunica sin cerrarse a una sola idea, pudiendo crear diversidad de opinión, comentando desde un punto totalmente periodístico, reflexionando sobre los medios y la cultura. En este trabajo se realiza un homenaje a esta personalidad intelectual del México contemporáneo quien, en la opinión de Karam, ha estudiado la comunicación desde varios puntos de vista. Se habla sobre su trayectoria y las obras que ha creado, así como todo el significado que se ha generado mediante éstas. El autor afirma que la obra de Monsiváis es generadora de un nuevo campo de reflexión que consiste en la vinculación entre lo social y la semiótica o semiosis, en la cual se conjugan tres procesos: la producción la creación y recepción del sentido.

Marta Rizo García, de la Universidad Autónoma de la Ciudad de México, es la autora de: Comunicología e interacción. El concepto de comunicación en el Interaccionismo Simbólico y la Escuela de Palo Alto. Uno de los puntos centrales que sostiene la autora es el concepto de la comunicación como interacción social. Retoma las aportaciones de la Escuela de Palo Alto y al Interaccionismo Simbólico para dar luz sobre el fenómeno de la comunicación. Señala que el punto de partida fundamental es la comprensión de las relaciones sociales mismas que se establecen por los participantes. Propone, Rizo, que la comunicación es el proceso básico de toda relación e indica que no es posible continuar en el supuesto de que el estudio de la comunicación se remita a una interacción simplista entre difusión y recepción.

Para cerrar esta segunda parte del XV AIC coneicc, Héctor Gómez Vargas de la Universidad Iberoamericana, León, escribe: Estratos espaciales y de comunicación en los estudios sobre la juventud. Una revisión de los estudios de los consumos culturales juveniles en México. En este trabajo se realiza una revisión bibliográfica y hemerográfica, sobre el consumo de los contenidos culturales que realizan los jóvenes en el país. Señala para ello que son tres las dimensiones que alimentan dicho consumo cultural: urbana, mediática y corporal. Gómez realiza un análisis exhaustivo de las corrientes teóricas y de las investigaciones empíricas realizadas en el contexto nacional sobre los jóvenes. Señala que hay dos cuestiones pendientes en la tarea de la comprensión del joven en la sociedad: 1) hacer más estudios del fenómeno de los grupos de jóvenes que logran una 
integración para entender mejor sus lógicas de congregación, de consumo de medios y de otros componentes culturales y 2) que estos estudios de recepción cultural se deben hacer mediante un enfoque socio-antropológico comprehensivo, que incorpore los desarrollos de apropiación de los espacios urbanos y los procesos de toma de identidad de los mismos jóvenes.

La tercera parte del XV AIC CONEICC: Investigación empirica de la comunicación, reúne tres trabajos que incluyen la búsqueda de evidencias empíricas para responder a sus preguntas de investigación y a sus hipótesis.

En su trabajo, Comunicación y migración juvenil: un imaginario de futuro en Tijuana, Gerardo León Barrios de la Universidad Autónoma de Baja California, Campus Tijuana, analiza cómo es que los jóvenes que han inmigrado a la ciudad fronteriza se ven obligados a reordenar sus anteriores formas de toma de identidad que llevaban a cabo en contextos rurales, semirurales o en otro tipo de centros urbanos. En esta ciudad, y ante sus instituciones sociales y públicas, los jóvenes, desde su individualidad y su capacidad de interacción, se ven en la necesidad de transformar sus estilos de vida y su cultura. El trabajo se sustenta en una investigación cualitativa cuyos sujetos de estudio son jóvenes inmigrantes en Tijuana. El autor argumenta elementos relacionados con la migración vinculados con: a) la decisión de migrar, b) la acción misma de emigrar y c) la reubicación en el nuevo espacio-territorio, tejen, con la nueva estructura social de este espacio-territorio, produciendo una nueva versión de vida sociocultural. León Barrios busca con su trabajo, vincular a las acciones individuales o micro a los sistemas sociales que se ubican en un nivel de análisis macro.

En el artículo, Comunicación intercultural y cultura laboral en una maquiladora trasnacional en Yucatán: un enfoque interdisciplinario, que realizan Verónica Soledad Garza Navejas y Gretty Guadalupe Escalante Góngora de la Universidad del Mayab, es posible degustar del análisis etnográfico, debidamente sustentado en un marco conceptual y referencial, de una fábrica de pantalón, ubicada en las cercanías de la ciudad de Mérida. El estudio se concentra en la cultura corporativa de la fábrica y en la comunicación intercultural que se da entre los integrantes de la misma. Las autoras concluyen que la cultura laboral que subsiste entre los empleados de esta organización es una que manifiesta una mezcla de dos componentes aparentemente contradictorios: a) la imposición de elementos culturales provenientes del corporativo que, en este caso, es una compañía norteamericana trasnacional y que tienen que ver propiamente con la actividad laboral de la planta, y b) el respeto de los componentes culturales de las operarias y operarios de la fábrica, mismos que se refieren a cuestiones 
de la cotidianeidad vital y social de los mismos, como son el tipo de alimento, la música, ente otros. Sugieren Garza y Escalante, que el logro de un auténtico diálogo intercultural solamente es posible en términos de igualdad. Para ello ven como necesaria la intervención del Estado en el establecimiento de políticas públicas pensadas para tal fin en el contexto de la penetración de capitales extranjeros en la región.

Por su parte, Rafael Tonatiuh Ramírez Beltrán de la Universidad Anáhuac México Norte, en su artículo, Representación social y formas de aproximación didácticas y éticas al cine por maestros: una exploración con palabras, creencias, estrategias e imágenes, realiza un trabajo de corte empírico cualitativo para apoyar los procesos de enseńanza aprendizaje de los valores, con el cine del siglo xxI.

A través de un estudio con el apoyo de entrevistas a profundidad, el autor descubre que un conjunto de maestros universitarios, señala que para facilitar algunos fundamentos de la ética, como son los valores y con el fin de propiciar el aprendizaje de algunas competencias, tales como la enseńanza para tomar decisiones, difícilmente se logra a través de métodos tradicionales. Estos maestros han encontrado que algunas películas facilitan que los educandos aprendan valores tales como la tolerancia, la libertad, la participación, la lealtad, entre otros, de situaciones y personajes en el film. Por otra parte, algunas competencias tales como aprender a tomar decisiones y a enfrentar conflictos, se pueden ejemplificar a través de la experiencia cinematográfica de los educandos. Ramírez concluye que su investigación le motiva a sistematizar las estrategias didácticas que permitan al profesor de las distintas materias a hacer un mejor uso del cine con fines educativos al interior de las aulas.

La cuarta parte del presente Anuario se titula Análisis simbólicos de fenómenos sociales y tiene como único artículo El culto a la Santa Muerte en el entramado simbólico de la sociedad del riesgo cuya autoría es de María Concepción Lara Mireles, de la Universidad Autónoma de San Luis Potosí. En este trabajo, la autora analiza la configuración de la narrativa social que se da a través de los rituales compartidos por los seguidores de la Santa Muerte y sus intercambios, mismos que contribuyen a la generación de sentido construido colectivamente. Además se analiza cómo ello se relaciona con las vivencias de la sociedad mexicana actual, que Lara describe como de incertidumbre y temor. Se relaciona esta crisis social a las situaciones de ilegalidad e inseguridad prevalecientes en México y cómo ello va transformando la cultura popular. La autora hace ver cómo el culto a la Señora de las Sombras, la Niña Blanca o la Santa Muerte, permite ver a sus seguidores a la misma muerte de frente y tener algún poder 
sobre ella a través de las prácticas rituales. De ahí que la Santa Muerte sea un fenómeno creado culturalmente como una forma liberadora del miedo.

La quinta parte del Anuario: Ecosistemas y nuevos actores de la comunicación, inicia con el artículo de Amaia Arribas Urrutia del Instituto Tecnológico y de Estudios Superiores de Monterrey, Campus Estado de México que tiene como título: El ecosistema virtual de los niños.

En este texto Arribas analiza la proliferación de evidencias empíricas existentes con respecto a las nuevas tecnologías de información y la gran aceptación que tienen en los hogares de todo el mundo. En particular, señala a las nuevas generaciones y específicamente a los niños y sus hábitos de consumo como una oleada que ha hecho que las empresas dediquen gran parte de su presupuesto publicitario a Internet. Por otra parte, la autora pone a la consideración la expansión de los mundos virtuales como espacios en los cuales los niños pueden tener experiencias más reales a través de plataformas 3D. Así mismo, en el trabajo se discute el recurso de las redes sociales, que vía Internet, permite a los adolescentes y jóvenes a tener una vida social más intensa mediante el intercambio de fotos, información personal, el chat y el uso de blogs. A la luz de estas reflexiones, Arribas advierte que el fenómeno de Internet se encuentra en sus albores, que los padres de familia pueden orientar a sus hijos en el uso adecuado de las posibilidades de interacción que esta tecnología ofrece, que el uso de la web ofrece un gran potencial para el entretenimiento, el desarrollo y la formación de los usuarios y para la interacción y la ampliación de la vida social de los individuos. Finaliza el trabajo con la reflexión de que niños y jóvenes son verdaderamente nativos digitales.

El segundo artículo de la quinta parte y último del XV Anuario de Investigación de la Comunicación CONEICC es: El prosumidor. El actor comunicativo de la Sociedad de la Ubicuidad y la autoría del mismo es de Octavio Islas Carmona del Instituto Tecnológico y de Estudios Superiores de Monterrey, Campus Estado de México. El título que ofrece Islas es sugerente de la posibilidad del hombre de estar en más de un lugar a la vez (ubicuidad), así como la de asimilar dos a más roles (prosumidor) a un mismo tiempo. A través de un análisis del pensamiento de los estudiosos de la información y de la comunicación en la era actual, quienes prefieren pensar el fenómeno de las nuevas tecnologías y su quehacer en la sociedad como la ecología cultural o la ecología mediática en la que habita el hombre en la actualidad, Islas pone bases firmes para repensar los conceptos fundamentales de la comunicación tales como los de emisor y receptor. Dichas propuestas implican, para los comunicadores, la posibilidad 
de repensar y replantear el mismo objeto de estudio de la comunicación. Con esta aportación de Islas, es posible vislumbrar los retos intelectuales y prácticos no en vías de manifestarse en la sociedad, sino presentes y desafiantes para la comunidad científica de la comunicación.

El Anuario cierra, como es tradición, con los Resúmenes de tesis ganadoras del Premio Nacional CONEICC 2007. Con ello se brinda la oportunidad a la comunidad de jóvenes investigadores, cuyos proyectos terminales de Licenciatura y de Maestría han sido calificados por el Comité de Evaluación de Tesis del CONEICC, como trabajos de excelencia.

En la Categoría de Licenciatura, la tesis ganadora de primer lugar, la realizó Alma Griscel Santiago Cuevas bajo la dirección del Mtro. Juan Carlos Ayala Perdomo ambos de la Universidad Autónoma del Estado de México. El título del trabajo es Mediaciones afectivas en el aula de clases: el afecto como herramienta de significación de los guiones del niño preescolar. El trabajo de investigación consiste en la búsqueda de las llamadas claves de razón práctica que ayudan a cumplir los procesos de enseñanza-aprendizaje en el aula, desde una perspectiva democrática, basada en el diálogo y la participación de cada miembro del grupo escolar. Rescata, la autora, el papel de la acción de los así llamados receptores con relación a su medio y a los guiones que construyen a partir del diálogo y de las interacciones que se dan entre los sujetos en las aulas del nivel preescolar.

El segundo lugar de la Categoría de Licenciatura le fue otorgado a Karina Berenice Bárcenas Barajas, de la Universidad Iberoamericana, León, con la tesis Educación artística y comunicación: escenarios en perspectiva desde la escuela primaria, que dirigió la Mtra. María de Lourdes Hernández Castañeda de la misma UIA, León. La autora argumenta que la escuela primaria posee características que la convierten en un escenario vasto y complejo para el estudio tanto de la comunicación como de los procesos de enseńanza-aprendizaje que ahí se desarrollan. Mediante su investigación, Bárcenas busca identificar los procesos de comunicación entre docentes y alumnos para entender cómo se desenvuelven, así como identificar, cómo es que se imparte la asignatura de educación artística en las mismas escuelas primarias. La realización de una investigación empírica, provee de evidencias a la autora para concluir que la comunicación en el aula, se da, efectivamente, a través del diálogo en todos los cursos analizados y ello es un factor que favorece a la educación artística en las escuelas primarias estudiadas.

Por su parte, el equipo integrado por Eugenia Galicia González, Marco Polo García Durán, Juan José Monroy Ramírez y Julio César Navarro Becerril, 
asesorados por las Mtras. Silvia Gutiérrez Vidrio y María del Carmen de Lara Rangel de la Universidad Autónoma Metropolitana, Unidad Xochimilco, presentan el trabajo: Las representaciones sociales del alcoholismo: una perspectiva de género, que obtuvo el tercer lugar en la Categoría de Licenciaturas. Los autores hacen ver que el consumo de alcohol ha permeado en la mayoría de los ámbitos sociales, debido a que los seres humanos siempre tienen motivaciones de todo tipo para ingerir alguna bebida etílica. La ingesta de alcohol, de acuerdo a los autores, se ha convertido en un medio de interacción con otros ámbitos que rodean a la persona: en lo social, en lo económico y cultural. En la opinión de los autores, con frecuencia se produce la enfermedad del alcoholismo entre quienes abusan de este tipo de bebidas. A través de un estudio cualitativo detectan que, en la percepción de sus sujetos de investigación, las mujeres alcohólicas son un blanco especialmente vulnerable de rechazo social debido a que se les califica cómo personas de poco carácter y que han perdido sus valores. Señalan que lejos de juzgar de manera preponderante a la mujer que sufre la enfermedad, se debe reconocer que el alcoholismo es un fenómeno transversal que afecta a ambos sexos, y que se da en grupos poblacionales de todas las edades, los niveles socio-económicos y afiliaciones religiosas.

Para concluir esta parte, se presenta el trabajo que realizó María Virginia Bon Pereira, del Instituto Tecnológico y de Estudios Superiores de Monterrey, Campus Monterrey, cuyo director de tesis es el Dr. José Carlos Lozano Rendón del mismo itesm, Campus Monterrey, que obtuvo el primer lugar de la Categoría de Maestría, con el título de: Contenidos y significados ideológicos en las once películas más taquilleras mundialmente. Mediante un estudio que incluyó el empleo de técnicas cuantitativas y cualitativas sobre las cintas más taquilleras del período 2001 y 2005, la autora señala que se trata de cintas de acción y aventuras producidas por las fábricas fílmicas que tienen su sede en Hollywood. Sus análisis, llevan la autora a concluir que las tramas casi invariablemente: a) llevan a la lucha ente los buenos y los malos; b) entre quienes se destaca casi invariablemente, a un personaje por su liderazgo, no siempre institucional, y c) que cuenta con seguidores cuyas acciones se manifiestan con escasos momentos de reflexión interna; d) que las tramas más referenciadas son el amor, la vida, la felicidad y el poder; e) que las historias generalmente se desenvuelven en espacios imaginarios y atemporales permitiendo la identificación con éstos por parte de los distintos públicos internacionales. Propone la autora que el cine, además de ser una fuente de entretenimiento evasivo, se convierta en un

$32 \cdot$ María Antonieta Rebeil Corella 
producto trascendente y que invite a los espectadores hacia mayores niveles de compromiso con ellos mismos y con la sociedad.

\section{RefLeXiones finALES}

A continuación se hacen algunas reflexiones acerca de las perspectivas de desarrollo que tiene el quehacer de la academia y la profesión de la comunicación en los próximos años.

Algunos de los estudiosos de la comunicación tienen la mirada puesta en la misma comunicación desde ciencias tales como la Sociología, la Antropología, la Política, la Economía, el Derecho, la Psicología, la Filosofía, entre otras, facilitando con ello, la comprensión de los fenómenos sociales que influyen en los procesos de la comunicación. Detrás de todo fenómeno hay personas que se ven afectadas por las decisiones y acciones comunicativas de otras personas. Los comunicadores estudian a las personas que se ven involucradas por los procesos e interacciones de la comunicación. La cuestión central de la comunicación nunca deja de ser las personas, independientemente del nivel de detalle con que se puedan analizar la complejidad de las mediaciones de la comunicación.

Algunas preguntas que permanecen sobre la mesa de discusiones que se derivan de las reflexiones realizadas por los autores del XV Anuario de Investigación de la Comunicación son:

- ¿Cuáles son los nuevos conceptos que se tienen que crear y definir para entender la comunicación dado los nuevos ambientes, actores y formas de interacción que aparecen en el actual ecosistema de la info-comunicación?

- ¿Cuáles son las vetas de investigación para analizar a la comunicación como portadora de sentido ético en la vida de las personas?

- En el marco del establecimiento de políticas públicas ¿cuál es el sentido que la ética de la comunicación debe tomar para orientar la toma de decisiones? ¿cuál es el papel de los investigadores de la comunicación en la conformación de estas políticas?

- ¿En qué medida se puede reorientar la producción de contenidos mediáticos de tal manera de que sean portadores de procesos de enseñanza-aprendizaje, de aquellas competencias para la vida que tienen que ver con el aprender a tomar decisiones, resolver conflictos sin violencia, promover la sana convivencia y la solidaridad entre los grupos humanos, la protección del medio ambiente, entre otras? 
- ¿Cuál es el sentido de que la información se haya convertido en un espectáculo y en una forma de entretenimiento que exacerba el morbo, la invasión de la privacidad y que atenta contra la dignidad de las personas?

- Hoy por hoy, la comunidad de investigadores de la comunicación se ha abocado al estudio de los grupos más vulnerables a las grandes transformaciones que ocurren en la sociedad: niños, jóvenes, mujeres, indígenas, obreros, migrantes, entre otros. ¿Cómo se puede establecer la corresponsabilidad entre los distintos actores que interactúan con estos grupos sociales y establecer las estrategias de comunicación de 360 grados involucrando a cada uno de estos actores según su nivel de participación?

- Si los procesos de comunicación intercultural suponen el intercambio en condiciones de igualdad entonces toca a los investigadores de la comunicación diseñar puentes de diálogo e interacción que asuman el reconocimiento de la dignidad del otro, del valor de su marco cultural, contextual, socioeconómico y simbólico. ¿Cuáles son las vertientes de la investigación de la comunicación en las organizaciones y fuera de éstas?

Un Anuario de Investigación de la Comunicación tiene la vocación de conducir a los pensadores hacia nuevos retos e innovadoras preguntas que posibiliten ir cubriendo la agenda pendiente en nuestro campo. Éste es el motor de la generación del conocimiento. Sobre la base de cuestionamientos acertados es que avanza el pensamiento y la búsqueda de la verdad. Sirvan estas palabras para agradecer a quienes contribuyen al avance de la construcción de la comunidad científica de la comunicación.

María Antonieta Rebeil Corella Coordinación de Investigación del ConeICC 